\title{
Comparisons between fresh and cryopreserved fat injections in facial lipofilling
}

\author{
Hyee Jae Yang, \\ Sang Yoon Kang \\ Department of Plastic Surgery, Kyung \\ Hee University Hospital, Kyung Hee \\ University College of Medicine, Seoul, \\ Korea
}

\begin{abstract}
Background: Autologous fat is considered an ideal filler material, and the use of cryopreserved fat grafts is promising in terms of flexibility and efficiency. Therefore, cryopreserved fat grafts have become more common in recent years; however, their complications require further consideration. Methods: We evaluated 53 patients who underwent facial lipofilling at our institution to confirm the clinical usefulness and safety of cryopreserved fat. Fresh fat injections with or without cryopreserved fat were administered. At one or more sites, 22 patients had a single fresh fat injection, four patients had two or more fresh fat injections, 16 patients had one fresh fat injection followed by one cryopreserved fat injection, six patients had one fresh fat injection followed by two cryopreserved fat injections, and five patients had two fresh fat injections and one or more cryopreserved fat injections.

Results: In total, 281 sets of injection procedures were performed at various sites, of which 170 involved one fresh fat injection, 89 involved one fresh fat injection and one cryopreserved fat injection, and 11 involved one fresh fat injection and two cryopreserved fat injections. One patient experienced self-resolving inflammation as a complication after the second injection in the right cheek. No statistically significant differences were found between the fresh and cryopreserved fat injections.

Conclusion: We suggest that cryopreserved fat is a useful and safe resource for multiple fat injections, with advantages including aseptic fat handling and the delicacy of the technique.
\end{abstract}

Keywords: Autografts / Cryopreservation / Fats

\section{INTRODUCTION}

Autologous fat injection (AFI) is performed widely in plastic surgery; it is used in cosmetic surgery for rejuvenation or volume augmentation of the face, breasts, and other parts of the body and in reconstructive surgery for the correction of contour

Correspondence: Sang Yoon Kang

Department of Plastic Surgery, Kyung Hee University Hospital, Kyung Hee

University College of Medicine, 23 Kyungheedae-ro, Dongdaemun-gu, Seoul

02447, Korea

E-mail: nuelk@nate.com

This article was presented as a poster at the Aesthetic Plastic Surgery 2017 on April $1-2,2017$, in Seoul, Korea

Received October 17, 2019 / Revised January 31, 2020 / Accepted February 3, 2020 deformities or the treatment of scar contracture or radiation injuries [1]. Autologous fat is considered to be an ideal filler material because it causes fewer adverse reactions than other fillers; furthermore, it is biocompatible because it is the patient's own fat. Harvesting autologous fat using liposuction is a comfortable, repeatable, and low-cost procedure for most patients [2].

However, the main problem associated with AFI for augmentation is the variable rate of absorption or necrosis at the grafted site. Transplant survival rates measured in terms of absorption or necrosis range from $30 \%$ to $80 \%$ [3]. In light of this variation, surgeons often perform overcorrection or repeated procedures, but patients may experience discomfort and dissatisfaction, an increased financial burden from the expenses associated with 
these procedures, and morbidity or trauma at the donor site. The remaining fat after the injection is usually discarded, which has spurred surgeons to explore ways of utilizing that fat. Accordingly, it has become increasingly common to utilize fat that has been cryopreserved after the initial fresh fat graft [4]. However, the fat that is cryopreserved after grafting is retained as dead and fibrous tissue, which may be associated with an elevated risk of clinical complications, such as oil cysts [5-7].

Very few clinical studies have investigated complications related to the use of cryopreserved fat. In this study, we present an analysis of our clinical results from facial lipofilling, with a comparison between fresh fat and cryopreserved fat injections.

\section{METHODS}

We analyzed the clinical records of patients who underwent facial lipofilling within the past 10 years and collected information about the injection site, the amount of fat injected, the number of fat injections, side effects, complications, and management. Fat injection-related symptoms included infections or inflammatory signs such as swelling, a palpable mass, or tenderness on the face. We excluded patients with a history of skin care procedures, such as chemical peeling or laser treatment, and dermatological skin lesions, such as acne. Sixty patients received fat injections. Fifty-one of these patients had no abnormal symptoms, while the remaining nine had inflammation or infections. Fifty-three of the 60 patients received fat injections at our institution, including seven men and 46 women. Their mean age was 48.6 years (range, 21-77 years). Seven patients were excluded from the statistical analysis because they were referred to our institution with fat injection-related symptoms, but did not receive the injections at our institution.

The study was approved by the Institutional Review Board of Kyung Hee University Hospital (IRB No. 2019-09-042-003) and performed in accordance with the principles of the Declaration of Helsinki. Written informed consent was obtained from patients.

\section{Surgical technique}

Fat was processed by manual harvesting using a tumescent fluid technique from the thigh or lower abdomen and centrifuged at 3,000 rpm for 3 minutes aseptically. We did not use cell-assisted lipo-transfer. Fat injections were performed using blunt Coleman infiltration cannulas (styles I and II) with the retrograde linear thread and fan technique. We ensured that the injected fat was spread evenly, without accumulating at a single point. After the injection, we applied an open dressing without compression and prescribed oral antibiotics and anti-inflammatory medi- cines for 5 days and oral prostaglandin for 14 days. For subsequent injections, we used cryopreserved fat, which was prepared by thawing. Except for the thawing step, the procedure for injecting cryopreserved fat was the same as the usual method. We used the cryopreserved fat within 6 months of frozen storage.

\section{Statistical analysis}

For statistical analyses, the Pearson chi-square test was performed to examine the associations between variables, and $p$ values $<0.05$ were considered to indicate statistical significance. Statistical analyses were performed using SPSS version 25.0 (IBM Corp., Armonk, NY, USA).

\section{RESULTS}

Twenty-two patients had a single fresh fat injection, four patients had two or more fresh fat injections, 16 patients had one fresh fat injection and one cryopreserved fat injection, six patients had one fresh fat injection and two cryopreserved fat injections, and five patients had two fresh fat injections and one or more cryopreserved fat injections (Table 1, Fig. 1).

Table 1. Type of fat injections at single sites

\begin{tabular}{lc}
\hline Type of fat injection & Number \\
\hline 1 Injection of fresh fat only & 22 \\
2 Or more injections of fresh fat only & 4 \\
1 Injection of fresh fat+1 injection of cryopreserved fat & 16 \\
1 Injection of fresh fat+2 injections of cryopreserved fat & 6 \\
2 Injections of fresh fat+1 or more injections of cryopreserved fat & 5 \\
\hline
\end{tabular}
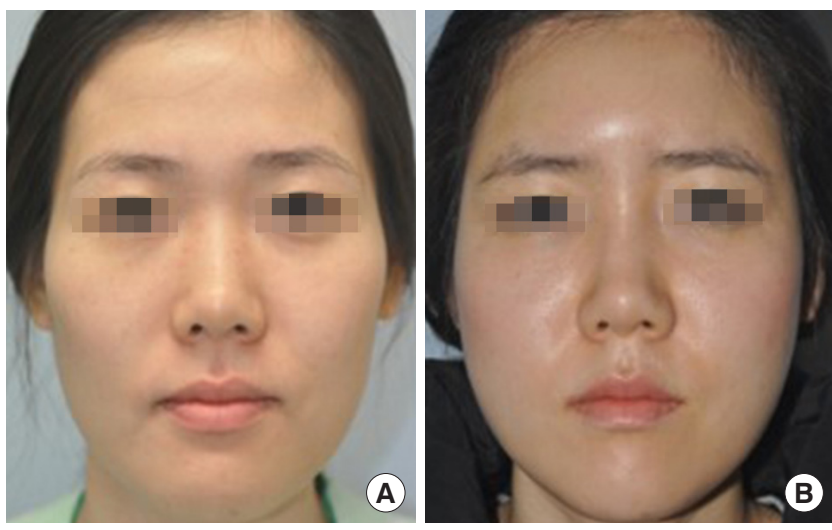

Fig. 1. (A) A 27-year-old woman with a flattened forehead, hollowed temporal area, sunken cheek, and low nasal dorsum. Fresh fat injections were performed in the forehead, both temporal areas, both lateral cheeks, and the nasal dorsum, with a total injection volume of $48 \mathrm{~mL}$. Two months later, another set of fresh fat injections, with a volume of $25 \mathrm{~mL}$, were performed in the same area. (B) Five months after fat injection, the hollowness on both sides of the lateral temporal area and cheek had disappeared. 
Overall, the most frequent site of fat injection was the nasolabial groove, followed in order by the cheek, forehead, and temples. However, the forehead was the most common site of injection in patients who received injections at only a single site. In total, 281 fat injections were performed, consisting of 170 fresh fat injections, 89 cryopreserved fat injections as the second injection, and 22 cryopreserved fat injections as the third injection. Complications occurred only in one patient, in whom signs of inflammation appeared after the second injection in the cheek, in which cryopreserved fat was injected (Table 2). The amount of fat injected by site and according to whether fresh or cryopreserved fat was used is shown in Table 3. The complication rate was $0.36 \%$ in all fat injections, $0.12 \%$ in second injections with cryopreserved fat, $0.90 \%$ in all cryopreserved fat injections, $3.57 \%$ in cryopreserved fat injections in the cheek, and $1.80 \%$ in fat injections. The mean interval between injections was 3.42 months (range, 1-8 months; data not shown). No complications occurred after fresh fat injections (the first injections). No statistically significant difference in the complication rate was found between patients who received only a fresh fat injection and those who received one fresh fat injection and one cryopreserved fat injection $(p=0.197)$. In the

Table 2. Number of fat injections by site

\begin{tabular}{|c|c|c|c|c|c|c|c|c|}
\hline Site & First injection & Second injection ${ }^{a)}$ & Third injectiona) & $\begin{array}{l}\text { Sum of second } \\
\text { and third injections }\end{array}$ & Total injections & Complications & Symptoms & $\begin{array}{l}\text { Average follow-up } \\
\text { (mo) }\end{array}$ \\
\hline Nasolabial groove & 48 & 24 & 7 & 31 & 79 & & & 14.4 \\
\hline Cheek & 40 & 21 & 7 & 28 & 68 & 1 & $\begin{array}{l}\text { Second injection; } \\
\text { tenderness, mass }\end{array}$ & 10.8 \\
\hline Forehead & 25 & 18 & 2 & 20 & 45 & & & 14.1 \\
\hline Temple & 26 & 9 & 3 & 12 & 38 & & & 13.9 \\
\hline Lower eyelid & 14 & 7 & 0 & 7 & 21 & & & 5.8 \\
\hline Upper eyelid & 8 & 3 & 0 & 3 & 11 & & & 7.1 \\
\hline Nose & 6 & 1 & 1 & 2 & 8 & & & 20.8 \\
\hline Jowl & 2 & 4 & 2 & 6 & 8 & & & 8.3 \\
\hline Jaw (chin) & 1 & 2 & 0 & 2 & 3 & & & 8.0 \\
\hline Sum & 170 & 89 & 22 & 111 & 281 & 1 & & \\
\hline
\end{tabular}

Bilateral fat injections in the nasolabial groove, cheek, temple, eyelid, and jowls were counted as two cases.

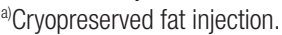

Table 3. The average amount of fat injections by site and counts

\begin{tabular}{|c|c|c|c|c|c|c|c|c|c|}
\hline Site & & $\begin{array}{c}\text { First } \\
\text { injectiona) }\end{array}$ & $\begin{array}{l}\text { Second } \\
\text { injection }^{b)}\end{array}$ & $\begin{array}{l}\text { Third } \\
\text { injection }^{\text {b) }}\end{array}$ & $\begin{array}{l}\text { Sum of second and third } \\
\text { injections }\end{array}$ & $\begin{array}{c}\text { Total } \\
\text { injections }\end{array}$ & $\begin{array}{c}\text { First } \\
\text { Min/Max }\end{array}$ & $\begin{array}{l}\text { Second } \\
\text { Min/Max }\end{array}$ & $\begin{array}{l}\text { Third } \\
\text { Min/Max }\end{array}$ \\
\hline \multirow[t]{2}{*}{ Nasolabial groove } & Left & 2.3 & 1.9 & 1.7 & 3.6 & 5.9 & $1.0 / 5.0$ & $0.5 / 4.5$ & $0.5 / 3.0$ \\
\hline & Right & 6.8 & 2.2 & 2.3 & 4.5 & 11.3 & $0.9 / 3.5$ & $0.9 / 5.5$ & $0.5 / 3.0$ \\
\hline \multirow[t]{2}{*}{ Cheek } & Left & 5.5 & 3.2 & 2.4 & 5.6 & 11.1 & $1.8 / 11.0$ & $1.5 / 8.0$ & $1.0 / 5.0$ \\
\hline & Right & 5.7 & 3.9 & 3.0 & 6.9 & 12.6 & $1.8 / 7.0$ & $1.5 / 7.0$ & $1.5 / 4.0$ \\
\hline Forehead & & 11.4 & 7.6 & 3.3 & 10.9 & 22.3 & $2.0 / 20.0$ & $1.1 / 16.0$ & $3.0 / 3.5$ \\
\hline \multirow[t]{2}{*}{ Temple } & Left & 5.9 & $2.0^{c)}$ & 3.0 & 5.0 & 10.9 & $1.0 / 20.0$ & $1.5 / 3.5$ & $2.5 / 3.0$ \\
\hline & Right & 5.6 & 2.9 & 2.8 & 5.6 & 11.2 & $1.0 / 14.0$ & $1.0 / 4.0$ & $1.0 / 5.0$ \\
\hline \multirow[t]{2}{*}{ Lower eyelid } & Left & 1.2 & 0.8 & - & - & 2.0 & $0.5 / 4.0$ & $0.5 / 1.2$ & - \\
\hline & Right & 0.9 & 0.7 & & & 1.6 & $0.5 / 2.2$ & $0.5 / 1.0$ & \\
\hline \multirow[t]{2}{*}{ Upper eyelid } & Left & 0.8 & 1.3 & - & - & 2.1 & $0.6 / 1.3$ & $1.0 / 1.5$ & - \\
\hline & Right & 0.8 & 1.0 & & & 1.8 & $0.5 / 1.3$ & $1.0 \mathrm{~d})$ & \\
\hline Nose & & 2.0 & 1.4 & 1.5 & 2.9 & 4.9 & $0.1 / 4.0$ & 1.4 & 1.5 \\
\hline \multirow[t]{2}{*}{ Jowl } & Left & 1.5 & 1.1 & 2.0 & 3.1 & 4.6 & $1.5^{d)}$ & $0.6 / 1.5$ & $2.0^{\circ)}$ \\
\hline & Right & 1.5 & 1.3 & 3.0 & 4.3 & 5.8 & $1.5^{\mathrm{d})}$ & $0.5 / 2.0$ & $3.0^{d)}$ \\
\hline Jaw (chin) & & 0.5 & 2.0 & - & - & 7.0 & 5.0 & 2.0 & - \\
\hline
\end{tabular}

Values are presented as values in cubic centimeters $(\mathrm{mL})$.

Min, minimum; Max, maximum.

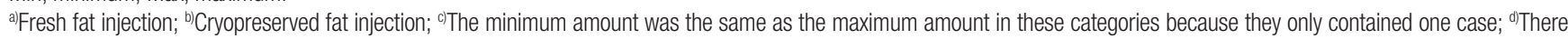
was only one case. 

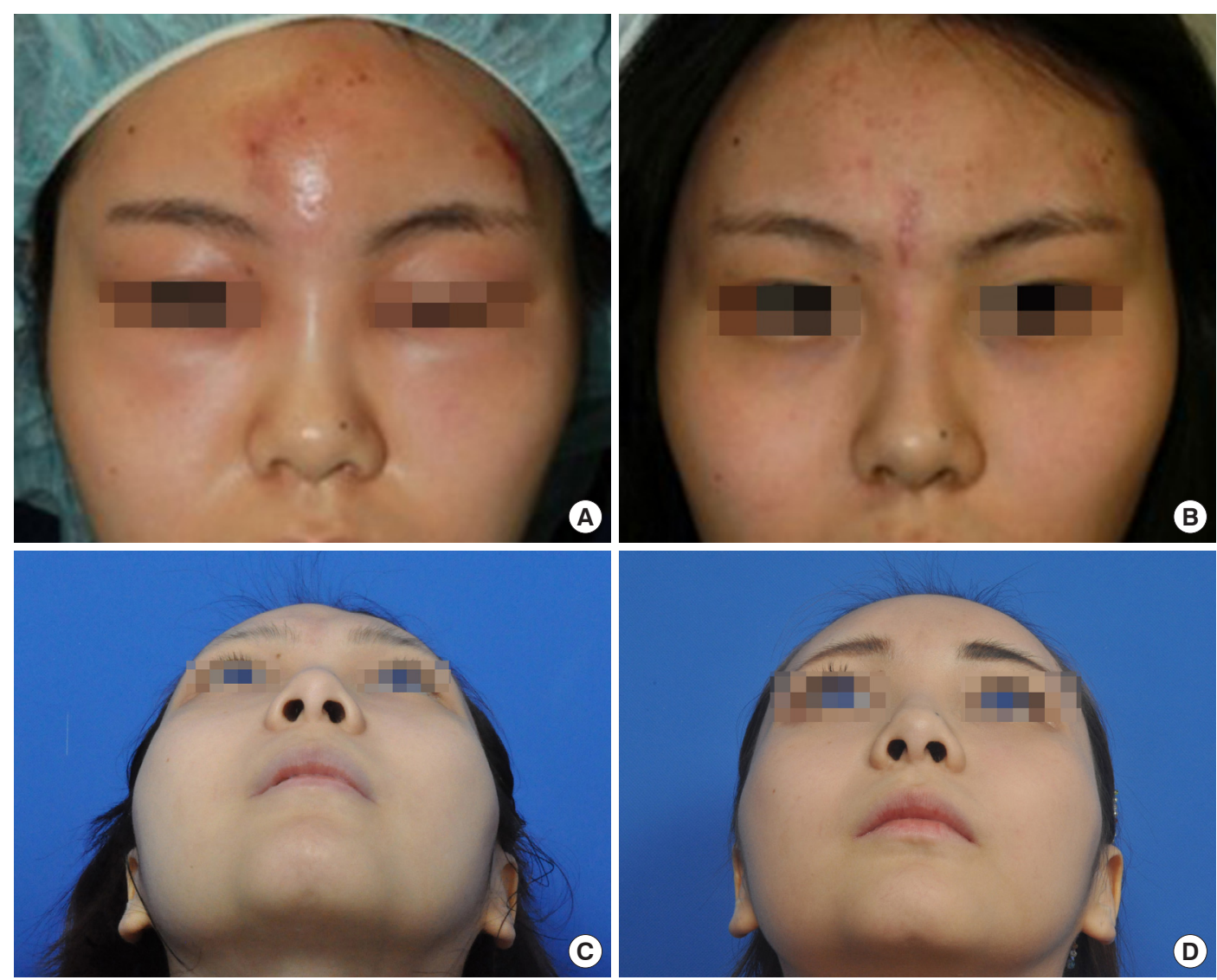

Fig. 2. (A) A 21-year-old woman with swelling, tenderness, and redness on the forehead and both upper eyelids and intermittent difficulty opening the eye at 1 week after receiving a cryopreserved fat injection in the forehead. After incision and curettage on the forehead and eyebrow, daily irrigation and curettage through the incision were performed for 3 weeks. (B) She had no recurrence of inflammation during a 5-month follow-up. (C) Sixteen months after treatment termination, a depression in the forehead was seen. (D) After the second set of autologous fat injections.

cheek, the complication rate was $1.47 \%$ of all fat injections, $4.76 \%$ of second injections, and $3.57 \%$ of cryopreserved fat injections. We found no statistically significant difference in the complication rate $(p=0.215)$ between patients who received one fresh fat injection and those who received second and third cryopreserved fat injections. Nine patients had inflammatory signs, of whom eight underwent surgical treatment, such as incision and drainage, curettage, and excision (Fig. 2). Of those patients, seven received a second injection containing cryopreserved fat, for a total of two injections at a 1- to 3-month interval, whereas the remaining patients received a cryopreserved fat injection at approximately a 1-month interval. The mean interval between the injections was 1.56 months.

\section{DISCUSSION}

In recent years, AFI has been performed widely in plastic surgery. Autologous fat is considered to be an ideal filler material because it causes fewer adverse reactions than other fillers; fur- thermore, since it is the patient's own fat, it is biocompatible. Harvesting autologous fat through liposuction is a comfortable, repeatable, and low-cost procedure for most patients [2]. The American Society of Plastic Surgeons organized a task force to assess the safety and efficacy of autologous fat grafting and reported it to be safe and to have a low risk of complications [8]. Common complications of AFI include patient dissatisfaction, acne, calcification, infection, skin necrosis, hematoma, paresthesia, and asymmetric or irregular contours. Blindness due to occlusion of the ophthalmic artery and cerebral infarction are serious complications that have been reported in some cases. However, the main obstacle associated with AFI for augmentation is the unpredictability of the outcomes due to variable, but high, absorption or necrosis rates at the grafted site [3]. Due to the difficulty of predicting the final outcome, surgeons often perform overcorrection or repeated procedures, which may cause patients to experience discomfort and dissatisfaction, an increased financial burden from medical expenses, and morbidity or trauma at the donor site. For these reasons, multiple 
uses of cryopreserved fat may elevate the possibility of recipient-site complications, such as low-grade inflammation, fat necrosis, and infection.

The risk of contamination is higher for cryopreserved fat than for fresh fat. Fresh fat can be contaminated during basic processes such as harvesting and centrifugation, but cryopreserved fat can also be exposed to contamination during additional processing steps such as freezing, storage, and thawing. Microorganisms can grow at low temperatures and in anaerobic environments during storage, causing infections at the grafted site after a fat injection; such infections are problematic, even if the causative microorganisms are of low virulence [9]. Furthermore, free oil can form and cryopreserved fat can leak as a result of intracellular ice formation. In 1972, Mazur et al. [10] reported that below the freezing point, pure crystalline watercontaining ice grows and the unfrozen fraction in the space between the growing ice contains all cells and solutes. As cooling continues, the volume of the unfrozen fraction decreases and its concentration increases. The increased osmotic pressure causes water to flow out of the cells. However, an appropriately slow cooling rate ensures sufficient water efflux from the intracellular environment, minimizing intracellular ice formation. Ultimately, the viscosity of the unfrozen fraction becomes too high for any crystals to form, and the unfrozen fraction turns into an amorphous state without ice crystals. However, a very slow cooling rate can cause total dehydration, which is lethal to the cells. In contrast, when the cooling rate is too fast, there is inadequate time for water to leave the cells and intracellular ice forms, which results in cell death in the cryopreserved fat [10].

Controversy persists regarding the viability and safety of cryopreserved fat. However, the use of appropriate medical protocols and cryopreservation techniques can ensure higher levels of viability and safety, with lower risks of complications than other surgical procedures [11-13]. Freezing, storage, and thawing are also essential processes when using cryopreserved fat. Many studies have been conducted to determine the best freezing, storage, and thawing conditions for appropriate medical protocols and cryopreservation techniques. As mentioned earlier, in the freezing process, an appropriate cooling rate is required to prevent intracellular ice formation, with a widely used rate being $1^{\circ} \mathrm{C} / \mathrm{min}$. Cells frozen at $-20^{\circ} \mathrm{C}$ were found to be more viable than those frozen at $-196^{\circ} \mathrm{C}$ [14]. Furthermore, the longer the storage period, the less viable cryopreserved fat is obtained after thawing [15]. In the thawing process, the recovery rate of antioxidant capacity and the neutralization of oxygen radicals may increase with the thawing temperature. Hwang et al. [16] reported that rapid thawing of cryopreserved fat in a water bath at $37^{\circ} \mathrm{C}$ maintained high mitochondrial activity and minimized cell damage.

Staphylococcus aureus is a common cause of inflammation in patients who undergo cosmetic skin surgery. However, in cases of chronically persistent lesions that are slowly reactive or nonreactive to conventional antibiotics, the presence of nontuberculous mycobacteria (NTM) must be considered. In addition, if inflammation does not occur immediately after fat injection, it is necessary to consider the possibility of infection with atypical mycobacteria such as Mycobacterium chelonae and M. abscessus, known as rapidly growing mycobacteria (RGM) [17]. These cause skin infections through skin wounds from invasive procedures such as injection, tattooing, and liposuction [18]. Nonspecific symptoms, including painful nodules, swelling, and abscess formation, appear in patients with RGM infections [19]. In addition to the difficulties posed by their nonspecific presentation, atypical mycobacterium infections are difficult to diagnose using conventional methods. Acid-fast bacilli staining has been reported to be positive for only $11 \%-27 \%$ of cases in a previous study [20]. Compared with other methods, molecular biology techniques such as polymerase chain reaction (PCR) may be useful because of their high sensitivity and accuracy [21]. NTM infection may be considered if a delayed infection occurs after an AFI and conventional antibiotics are ineffective. Antibiotic sensitivity tests using empirical antibiotics and diagnostic methods such as PCR should be performed to diagnose an NTM infection [22]. If the treatment response is slow or no improvement is observed, surgical procedures such as debridement and excision might also be useful [23].

In the present cases, patients with signs of an acute infection were treated surgically. They experienced no recurrence and received an additional autologous fat graft for contour recovery. Surgeons who perform fat injections must keep in mind that lesions showing inflammatory signs after a fat injection will have a complicated clinical course. Moreover, although it was difficult to compare the referred cases and our cases, a marked difference was found between these groups in the interval between injections (1.56 months vs. 3.53 months). The survival rate generally stabilizes 3 months after the graft [24-28]. Furthermore, a longer interval is suitable for promoting the survival of previously inserted fat and recovery from the inflammatory environment induced by the fat injection. In addition, a large graft volume in a small area will show a lower survival rate compared than a small amount grafted in a large recipient area [29]. It has been reported that in Asians, a relatively large amount of fat must be injected into the forehead to create a thick blanket of fat for esthetic purposes, and under such circumstances, the anoxic central core of the grafted fat might be larger. The cheek looks like a basin and has a retaining ligament, the zygomatico- 
cutaneous ligament (also known as McGregor's patch) [30]. The small spot at the center of the depressed area requires much more fat to be grafted than the peripheral area, while the retaining ligament grabs the skin, preventing outward expansion. These factors may cause surgeons to overlook the effect of a graft or the presence of a new dimple, resulting in a tendency to perform overinjections, which carries the risk of complications. In any case, critical factors in micro-fat grafting include the relocation of mature adipocytes to the perfusion-rich layer and reduction of the anoxic central core fragment, resulting in failure of the apoptotic pathway [31]. We found no significant differences in the complication rate according to whether fresh or cryopreserved fat was used for AFI, indicating that careful handling and adequate technique protect from the anticipated complications of fat injection using cryopreserved fat.

A limitation of our study is its relatively small sample size. Moreover, it is necessary to study the relationships between complications and the interval of fat injections in patients who received facial lipofilling with a fresh fat injection followed by a cryopreserved fat injection on an atypical schedule.

Regarding the technique, elimination of free fat oil and nonviable components through centrifugation or mesh techniques may be useful for decreasing resorption [32] and the incidence of foreign body reactions. Furthermore, a blunt cannula, not a sharp one, should be used. Pre-tunneling and fat injection should be carefully and gently performed to prevent vessel damage because performing these procedures without a suitably delicate technique can cause fat embolism, resulting in serious complications such as blindness and tissue necrosis [9].

All injectable fillers cause normal foreign body reactions, which may develop into a foreign body granuloma in some patients. Depending on the injectable filler material, the histological reaction and permanence differ. The following five types of fillers are distinguished: autologous fat, natural fillers such as collagen and hyaluronic acid, fluid fillers such as fluid silicone and acrylamides, particulate materials such as polymethylacrylate, and microspheres such as resorbable dextran and polymethylmethacrylate [33]. As the immune response to various filler materials differs, and it remains unknown whether they cause late inflammation or granuloma formation, patients should be informed about the benefits and risks of cryopreserved fat injections, even autologous fat grafts, when choosing the appropriate fat injection technique.

In summary, clinical reports on the results of cryopreserved fat injections are rare. Our findings reveal no significant differences in the clinical outcomes of fat injections according to the use of fresh fat or cryopreserved fat. Accordingly, cryopreserved fat is a useful and safe resource for multiple fat injections, with advantages including aseptic fat handling and the delicacy of the technique.

\section{NOTES}

\section{Conflict of interest}

No potential conflict of interest relevant to this article was reported.

\section{Ethical approval}

The study was approved by the Institutional Review Board of Kyung Hee University Hospital (IRB No. 2019-09-042-003) and performed in accordance with the principles of the Declaration of Helsinki. Written informed consents were obtained.

\section{Patient consent}

The patients provided written informed consent for the publication and the use of their images.

\section{ORCID}

Hyee Jae Yang https://orcid.org/0000-0001-8068-8770

Sang Yoon Kang https://orcid.org/0000-0002-6299-6371

\section{REFERENCES}

1. Mashiko T, Wu SH, Feng J, Kanayama K, Kinoshita K, Sunaga A, et al. Mechanical micronization of lipoaspirates: squeeze and emulsification techniques. Plast Reconstr Surg 2017;139: 79-90.

2. Coleman SR. Structural fat grafts: the ideal filler? Clin Plast Surg 2001;28:111-9.

3. Guerrerosantos J, Gonzalez-Mendoza A, Masmela Y, Gonzalez MA, Deos M, Diaz P. Long-term survival of free fat grafts in muscle: an experimental study in rats. Aesthetic Plast Surg 1996;20:403-8.

4. Ohashi M, Chiba A, Nakai H, Fukuda E, Higuchi T. Serial injections of cryopreserved fat at $-196^{\circ} \mathrm{C}$ for tissue rejuvenation, scar treatment, and volume augmentation. Plast Reconstr Surg Glob Open 2018;6:e1742.

5. Mashiko T, Wu SH, Kanayama K, Asahi R, Shirado T, Mori M, et al. Biological properties and therapeutic value of cryopreserved fat tissue. Plast Reconstr Surg 2018;141:104-15.

6. Lee S, Lee JH, Choi HS. Clinical characteristics of patients with a periorbital mass after autologous fat injection for facial augmentation and short-term outcomes of steroid treatment. J Plast Reconstr Aesthet Surg 2015;68:1498-503.

7. Kim SM, Kim YS, Hong JW, Roh TS, Rah DK. An analysis of the experiences of 62 patients with moderate complications af- 
ter full-face fat injection for augmentation. Plast Reconstr Surg 2012;129:1359-68.

8. Gutowski KA; ASPS Fat Graft Task Force. Current applications and safety of autologous fat grafts: a report of the ASPS fat graft task force. Plast Reconstr Surg 2009;124:272-80.

9. Park JW. Ocular swelling after forehead fat graft. Arch Aesthetic Plast Surg 2014;20:85-91.

10. Mazur P, Leibo SP, Chu EH. A two-factor hypothesis of freezing injury: evidence from Chinese hamster tissue-culture cells. Exp Cell Res 1972;71:345-55.

11. Pu LL, Cui X, Fink BF, Gao D, Vasconez HC. Adipose aspirates as a source for human processed lipoaspirate cells after optimal cryopreservation. Plast Reconstr Surg 2006;117:1845-50.

12. Khouri RK Jr, Khouri RK. Current clinical applications of fat grafting. Plast Reconstr Surg 2017;140:466e-486e.

13. Rigotti G, Marchi A, Galie M, Baroni G, Benati D, Krampera M, et al. Clinical treatment of radiotherapy tissue damage by lipoaspirate transplant: a healing process mediated by adiposederived adult stem cells. Plast Reconstr Surg 2007;119:1409-22.

14. MacRae JW, Tholpady SS, Ogle RC, Morgan RF. Ex vivo fat graft preservation: effects and implications of cryopreservation. Ann Plast Surg 2004;52:281-2.

15. $\mathrm{Pu}$ LL. Cryopreservation of adipose tissue. Organogenesis 2009;5:138-42.

16. Hwang SM, Lee JS, Kim HD, Jung YH, Kim HI. Comparison of the viability of cryopreserved fat tissue in accordance with the thawing temperature. Arch Plast Surg 2015;42:143-9.

17. Dessy LA, Mazzocchi M, Fioramonti P, Scuderi N. Conservative management of local Mycobacterium chelonae infection after combined liposuction and lipofilling. Aesthetic Plast Surg 2006;30:717-22.

18. Brown-Elliott BA, Wallace RJ Jr. Clinical and taxonomic status of pathogenic nonpigmented or late-pigmenting rapidly growing mycobacteria. Clin Microbiol Rev 2002;15:716-46.

19. Bartralot R, Garcia-Patos V, Sitjas D, Rodriguez-Cano L, Mollet J, Martin-Casabona N, et al. Clinical patterns of cutaneous nontuberculous mycobacterial infections. Br J Dermatol 2005; 152:727-34.

20. Bartralot R, Pujol RM, Garcia-Patos V, Sitjas D, Martin-Casabona N, Coll P, et al. Cutaneous infections due to nontuberculous mycobacteria: histopathological review of 28 cases. Comparative study between lesions observed in immunosuppressed patients and normal hosts. J Cutan Pathol 2000;27:124-9.

21. Abdalla CM, de Oliveira ZN, Sotto MN, Leite KR, Canavez
FC, de Carvalho CM. Polymerase chain reaction compared to other laboratory findings and to clinical evaluation in the diagnosis of cutaneous tuberculosis and atypical mycobacteria skin infection. Int J Dermatol 2009;48:27-35.

22. Kim SK, Choi JA, Kim MH, Kim MS, Lee KC. Treatment of the Mycobacterium chelonae infection after fat injection. Arch Plast Surg 2015;42:68-72.

23. Chang $\mathrm{CH}$, Chang YY, Lu PH. Non-tuberculous mycobacteria infection following autologous fat grafting on the face. Aesthet Surg J 2017;38:NP1-5.

24. Wang L, Luo X, Lu Y, Fan ZH, Hu X. Is the resorption of grafted fat reduced in cell-assisted lipotransfer for breast augmentation? Ann Plast Surg 2015;75:128-34.

25. Sasaki GH. The safety and efficacy of cell-assisted fat grafting to traditional fat grafting in the anterior mid-face: an indirect assessment by 3D imaging. Aesthetic Plast Surg 2015;39:833-46.

26. Chang Q, Li J, Dong Z, Liu L, Lu F. Quantitative volumetric analysis of progressive hemifacial atrophy corrected using stromal vascular fraction-supplemented autologous fat grafts. Dermatol Surg 2013;39:1465-73.

27. Schendel SA. Enriched autologous facial fat grafts in aesthetic surgery: 3D volumetric results. Aesthet Surg J 2015;35:913-9.

28. Dos Anjos S, Matas-Palau A, Mercader J, Katz AJ, Llull R. Reproducible volume restoration and efficient long-term volume retention after point-of-care standardized cell-enhanced fat grafting in breast surgery. Plast Reconstr Surg Glob Open 2015; 3:e547.

29. Khouri RK, Rigotti G, Cardoso E, Khouri RK Jr, Biggs TM. Megavolume autologous fat transfer: part I. theory and principles. Plast Reconstr Surg 2014;133:550-7.

30. Furnas DW. The retaining ligaments of the cheek. Plast Reconstr Surg 1989;83:11-6.

31. Suga H, Eto H, Aoi N, Kato H, Araki J, Doi K, et al. Adipose tissue remodeling under ischemia: death of adipocytes and activation of stem/progenitor cells. Plast Reconstr Surg 2010;126: 1911-23.

32. Salinas HM, Broelsch GF, Fernandes JR, McCormack MC, Meppelink AM, Randolph MA, et al. Comparative analysis of processing methods in fat grafting. Plast Reconstr Surg 2014; 134:675-83.

33. Lemperle G, Morhenn V, Charrier U. Human histology and persistence of various injectable filler substances for soft tissue augmentation. Aesthetic Plast Surg 2003;27:354-66. 